\title{
latrogenia medicamentosa em idosos hospitalizados no interior do Amazonas
}

\author{
Iatrogenic medication in elderly hospitalized in Amazonas
}

interior

Iatrogenia de drogas en ancianos hospitalizados en el interior

de Amazonas

Andriele Valentim da Costa

Luana de Melo Lisboa

Deyvylan Araújo Reis

Hyana Kamila Ferreira de Oliveira

RESUMO: As iatrogenias são ações decorrentes de intervenções médicas, ou de membro/s de uma equipe médica, que resultem em consequências indesejáveis ou prejudiciais à saúde de um indivíduo hospitalizado. O objetivo deste estudo foi identificar iatrogenias medicamentosas que ocorreram com pacientes idosos internados no Hospital Regional da cidade de Coari, estado de Amazonas, Brasil. Os dados foram coletados de 100 prontuários, dos quais se identificou, por meio de um instrumento criado pelos autores, que a omissão de doses foi a iatrogenia mais frequente em $83,0 \%$ dos casos, recomendando-se que se busque compreender causas, fatores de risco, consequências, e pensar em estratégias para diminuir ou minimizar essas ocorrências.

Palavras-chave: Doença iatrogênica; Idoso; Enfermagem.

ABSTRACT: Iatrogenies are actions resulting from medical interventions or from their staff, resulting in undesirable or harmful consequences to the health of the hospitalized individual. The objective of this study was to identify the drug iatrogenies that occurred with elderly patients hospitalized at the Regional Hospital of Coari, Amazonas. The data were collected from 100 charts, which identified that through an instrument created by the authors. The absence of the dose was more frequent of iatrogeny with $83,0 \%$.

Keywords: Iatrogenic Disease; Aged; Nursing. 
RESUMEN: Las iatrogenias son acciones derivadas de intervenciones médicas o de su equipo, que resulten em consecuencias indeseables o perjudiciales para la salud del individuo hospitalizado. El objetivo de este estúdio fue identificar las iatrogenias medicamentosas que ocurrieron con pacientes ancianos internados em el Hospital Regional de Coari, Amazonas. Los datos fueron recolectados de 100 prontuarios, que identifico que por medio de um instrumento creado por los autores. La omisión de dosis fu ela iatrogenia más frecuente com el 83,0\%.

Palabras clave: Enfermedad iatrogénica; Anciano; Enfermería.

\section{Introdução}

As instituições de saúde têm, como um dos princípios básicos, o fornecimento de bens e serviços com o mínimo, ou até a ausência total de riscos e falhas. No entanto, a ocorrência de iatrogenias coloca em discussão a segurança de um paciente e levanta a questão da qualidade dos serviços de saúde (Padilha, 2006).

As iatrogenias podem ser definidas como ações decorrentes de intervenções médicas ou de uma equipe médica, que resultem em consequências indesejáveis ou prejudiciais à saúde de um paciente (Carvalho-Filho, et al., 1998; Padilha, 2006). Este termo "iatrogenia" vem sendo substituído por "evento adverso"; no entanto, na prática geriátrica a palavra iatrogenia e/ou doença iatrogênica ainda são termos frequentemente usados (Szlejf, 2010).

Os eventos iatrogênicos têm origem multifatorial e configuram-se como um grande desafio para a saúde pública (Diniz, 2010). Segundo Schmidt, et al. (2011), esses eventos contribuem para uma série de prejuízos para o sistema de saúde, dado que envolvem o aumento de custos referentes ao tratamento e o prejuízo direto ao paciente, como o aumento do sofrimento, sequelas ou até mesmo o óbito.

As iatrogenias estão relacionadas ao uso indevido de medicamentos, à execução inadequada de procedimentos, ou à omissão dos cuidados prestados à saúde. Entre as principais ações iatrogênicas, destacam-se: uso excessivo de medicamentos, imposições dietéticas, quedas, cirurgias desnecessárias, e surgimento de lesão por pressão (Matos, et al., 2011).

Como o aumento da população idosa é um fenômeno mundial que vem ocorrendo tanto em países desenvolvidos como em desenvolvimento, cujas mudanças demográficas vêm sendo acompanhadas de alterações epidemiológicas, caracterizadas pelo predomínio de doenças crônicas, a preocupação com a iatrogenia medicamentosa em idosos tem se tornado crescente, pois a variedade de doenças crônicas leva a uma maior demanda do uso contínuo e simultâneo de medicamentos, 
contribuindo para a ocorrência de erros na indicação da terapêutica farmacológica, para a prescrição de medicamentos inapropriados, e para a polifarmácia (Correr, Pontarolo, Ferreira, \& Baptistão, 2007; Guiselli, Ely, Engroff, Nogueira, \& Gomes, 2016).

Quando se trata de idosos, a iatrogenia tem maior impacto, pois as modificações determinadas pelo envelhecimento, pluripatologia, a maior frequência de procedimentos diagnósticos, o uso frequente de medicamentos inclusive associados, maior tempo de internação, a utilização de métodos terapêuticos mais agressivos e sofisticados, podem contribuir para o aumento da incidência de iatrogenia na pessoa idosa (Carvalho, et al., 1998; Szlejf, et al., 2008).

O registro das complicações iatrogênicas tem sido pouco evidente e vago, sendo poucos os serviços de saúde que adotam protocolos para a verificação dessas complicações; além disso, se torna difícil identificar esses eventos iatrogênicos quando os próprios profissionais tentam encobri-los (Carvalho-Filho, et al., 1998; Madalosso, 2000). De acordo com Padilha (2001), deve existir um olhar crítico e investigativo sobre as falhas, não com o objetivo de punição, mas, sim, a fim de compreender as causas e trabalhá-las de forma eficaz para beneficiar o cliente e todos os envolvidos no processo de assistência à saúde.

O interesse por esta investigação surgiu pelo fato de os estudos relacionados a esse assunto ainda serem escassos, principalmente em âmbito nacional, havendo poucas publicações em base de dados confiáveis principalmente envolvendo a pessoa idosa. Dessa forma, este trabalho tem como objetivo identificar iatrogenias medicamentosas que ocorreram com pacientes idosos internados no Hospital Regional de Coari, Amazonas.

\section{Método}

Trata-se de um estudo descritivo, transversal, documental, retrospectivo, com abordagem quantitativa. Os sujeitos do estudo foram pacientes idosos com idade igual ou superior a 60 anos, que foram internados na clínica médica do Hospital Regional de Coari, Amazonas, no período de janeiro a junho de 2018. Conforme os critérios de inclusão, a coleta de dados aconteceu através da análise de 100 prontuários de pacientes internados. A obtenção dos dados foi realizada no setor de estatística da instituição supracitada, durante os meses de agosto a novembro de 2018.

O instrumento para a coleta foi um formulário construído pelos autores, contendo questões com características demográficas e socioeconômicas (idade, sexo, situação de trabalho) e dados da internação hospitalar (doença crônica, causa da internação, dias de internação, número de medicamentos utilizados durante a internação, nome e classe dos medicamentos prescritos, ocorrências iatrogênicas medicamentosas e agravos relacionados à iatrogenia medicamentosa), contendo 11 questões que nortearam os pesquisadores na coleta dos dados. 
As informações extraídas dos prontuários foram realizadas de maneira minuciosa através das evoluções e registros de enfermagem, além dos dados sobre os sinais vitais, prescrições médicas e horários de aprazamento dos medicamentos, procurando identificar omissão de doses, doses inapropriadas, via de administração imprópria, medicação errada, horário de administração errado, e se houve o registro de algum tipo de agravo relacionado com esses eventos. Os dados coletados foram tabulados e analisados por meio do programa estatístico Statistical Package for the Social Sciences (SPSS), versão 20.0.

Esta pesquisa foi aprovada pelo Comitê de Ética em Pesquisa da Universidade Federal do Amazonas, número do CAAE 84450418.6.0000.5020, sob o parecer n. ${ }^{\circ}$ 2.545.875. Para submeter ao Comitê de Ética, foi necessário o termo de anuência da Secretaria de Saúde, autorizando a realização da pesquisa. Obteve-se dispensa do Termo de Consentimento Livre e Esclarecido (TCLE), por se tratar de um estudo com dados secundários, não envolvendo a participação direta dos pacientes.

\section{Resultados}

Foram analisados 100 prontuários; destes, 46 (46,0\%) eram de mulheres; e $54(54,0 \%)$ de homens; $25(28,2 \%)$ com idade entre 60 a 69 anos; $40(37,6)$ com idade entre 70 a 79 anos; e 35 $(34,2 \%)$ com 80 anos ou mais; com média de 75,7( $\pm 9,11)$ anos. Apenas $60(60,0 \%)$ dos idosos recebiam aposentadoria. A média de idade para os homens foi de 75,9 $( \pm 9,2)$ anos; e mediana de 75; para as mulheres foi de $74,9( \pm 8,9)$ anos mediana de 75 . Em relação aos dados ocupacionais, quatro $(67,0 \%)$ eram de agricultores; dois $(22,5 \%)$ comerciantes; um vendedor $(10,5 \%)$. Quanto ao número de medicamentos utilizados durante a internação, $64(64,0 \%)$ idosos fizeram uso de igual ou superior a 10 medicamentos por dia.

Tabela 1. Distribuição das características sociodemográficos e número de medicamentos prescritos por dia, Coari, Amazonas

\begin{tabular}{|l|l|l|l|l|}
\hline \multicolumn{1}{|c}{ Variável } & $\mathrm{N}$ & $\%$ & Média & Desvio padrão \\
\hline Sexo & 46 & 46,0 & & \\
\hline Feminino & 54 & 54,0 & & \\
Masculino & & & & \\
\hline Faixa etária & 25 & 28,2 & & \\
\hline 60 a 69 anos & 40 & 37,6 & 75,7 & \\
70 a 79 anos & 35 & 34,2 & & \\
$\geq 80$ anos & & & & \\
\hline Aposentado & 60 & 60,0 & & \\
\hline Sim & 40 & 40,0 & & \\
Não & & & & \\
\hline
\end{tabular}




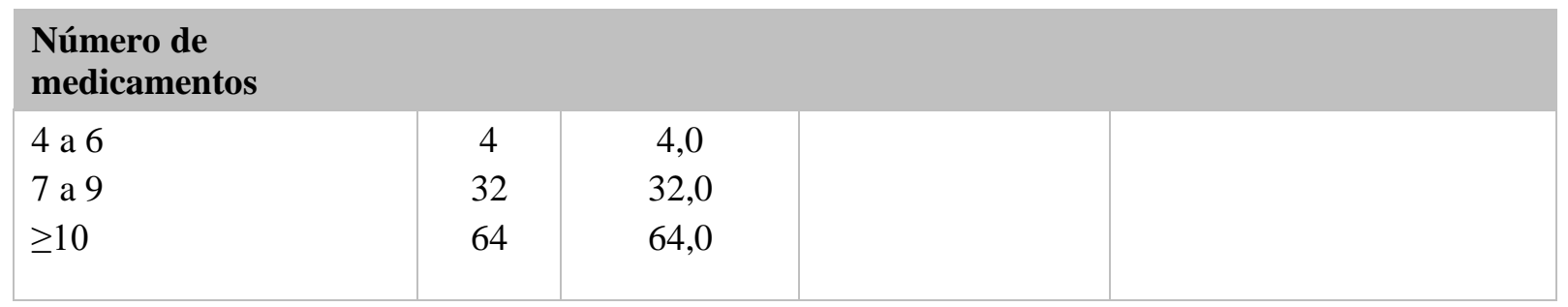

Fonte: Dados da pesquisa, 2018

Foram identificados 242 diagnósticos médicos que levaram à internação; destes, o mais frequente foi de Pneumonia ( 24 em homens e 19 em mulheres, totalizando 43 casos).

Os diagnósticos médicos nesta pesquisa foram classificados conforme a Classificação Internacional de Doenças (CID-10). De acordo com esta classificação, as doenças mais frequentes foram representadas por aquelas do trato respiratório $(21,9 \%)$, seguidas de doenças do aparelho circulatório $(11,2 \%)$.

Tabela 2. Diagnóstico médico de internação conforme a Classificação Internacional de Doenças (CID 10), Coari, AM

\begin{tabular}{|c|c|c|}
\hline & $\mathbf{N}$ & $\%$ \\
\hline Doenças do aparelho respiratório & 53 & 21,9 \\
\hline Doença do aparelho circulatório & 27 & 11,2 \\
\hline Doenças do aparelho geniturinário & 22 & 9,1 \\
\hline Doença do aparelho digestivo & 13 & 5,4 \\
\hline Algumas doenças infecciosas e parasitárias & 8 & 3,3 \\
\hline Doença da pele e do tecido subcutâneo & 6 & 2,5 \\
\hline $\begin{array}{l}\text { Sintomas, sinais e achados anormais de } \\
\text { exames clínicos e de laboratório, não } \\
\text { classificados em outra parte. }\end{array}$ & 6 & 2,5 \\
\hline $\begin{array}{l}\text { Doença do sangue e dos órgãos } \\
\text { hematopoiéticos e alguns transtornos } \\
\text { imunitários }\end{array}$ & 5 & 2,1 \\
\hline $\begin{array}{l}\text { Doenças endócrinas, nutricionais e } \\
\text { metabólicas. }\end{array}$ & 4 & 1,7 \\
\hline $\begin{array}{l}\text { Lesões, envenenamentos e algumas outras } \\
\text { consequências de causas externas }\end{array}$ & 2 & 0,8 \\
\hline Doenças do sistema nervoso. & 1 & 0,4 \\
\hline Total & 147 & 60,7 \\
\hline
\end{tabular}

Fonte: Dados da pesquisa, 2018

Dentre os idosos, $73(73,0 \%)$ possuíam alguma doença de base registrada, variando de uma a quatro doenças por paciente. A Hipertensão Arterial Sistêmica (HAS) destaca-se pela frequência com que foi observada, num total de 49 casos (48,0\%), seguida pela Diabetes Mellitus (DM) teve uma frequência de 35 casos $(34,3 \%)$. 
Tabela 3. Distribuição das doenças preexistentes, Coari, AM

\begin{tabular}{l|c|c|} 
Doença pré-existente & N & \% \\
\hline Hipertensão arterial sistêmica & 49 & 48 \\
\hline Diabetes mellitus tipo 2 & 35 & 34,3 \\
\hline $\begin{array}{l}\text { Doença pulmonar obstrutiva } \\
\text { crônica }\end{array}$ & 3 & 2,9 \\
\hline Hepatopatia & 3 & 2,9 \\
\hline $\begin{array}{l}\text { Insuficiência cardíaca } \\
\text { congestiva }\end{array}$ & 2 & 2 \\
\hline Outros & 10 & 9,9 \\
\hline Total & 102 & 100 \\
\hline
\end{tabular}

Fonte: Dados da pesquisa, 2018

Na tabela 4, consta a relação entre os dias de internação e o número de eventos iatrogênicos. Os dias de internação tiveram como mínimo um dia, e como máximo 60 dias, com média de 10 ( \pm 1,0) dias. O tempo médio de internação, para os homens, foi de $9,0( \pm 6,8)$ e mediana de 7,0 ; para as mulheres, foi de $10,2( \pm 9,9)$ e mediana de 7,0 .

Tabela 4. Relação dos dias de internação e o número de eventos iatrogênicos, Coari, AM

\begin{tabular}{c|c|c|c|c|c|}
\hline $\begin{array}{c}\text { Dias de } \\
\text { internação }\end{array}$ & \multicolumn{5}{c}{$\mathbf{N}^{\mathbf{0}}$ Eventos Iatrogênicos } \\
\hline & $\mathrm{N}(\%)$ & $\mathrm{N}(\%)$ & $\mathrm{N}(\%)$ & $\mathrm{N}(\%)$ & Total \\
\hline & $\mathbf{1}$ à $\mathbf{3}$ & $\mathbf{4}$ à $\mathbf{6}$ & $\mathbf{7}$ à $\mathbf{9}$ & $\geq \mathbf{1 0}$ & 14 \\
\hline $\mathbf{1}$ à $\mathbf{3}$ & $10(14,7)$ & $1(4,2)$ & $2(50,0)$ & $1(25,0)$ & 33 \\
\hline $\mathbf{4}$ à $\mathbf{6}$ & $20(29,4)$ & $11(45,8)$ & $2(50,0)$ & $0(0,0)$ & 23 \\
\hline $\mathbf{7}$ à $\mathbf{9}$ & $18(26,5)$ & $5(20,8)$ & $0(0,0)$ & $0(0,0)$ & 30 \\
\hline$\geq \mathbf{1 0}$ & $20(29,4)$ & $7(29,2)$ & $0(0,0)$ & $3(75,0)$ & 100 \\
\hline Total & $68(100)$ & $24(100)$ & $4(100)$ & $4(100)$ & \\
\hline
\end{tabular}

Fonte: Dados da pesquisa, 2018

Das iatrogenias medicamentosas identificadas nos registros, a omissão de doses foi a mais frequente, estando presente em $83(83,0 \%)$ internações, seguida da medicação errada com $12(12,0 \%)$. Foi considerada omissão de doses aquelas que constavam na prescrição, no entanto não houve registro da administração. 
Tabela 5. Iatrogenias medicamentosas, Coari, AM

\begin{tabular}{|c|c|c|}
\hline Iatrogenias medicamentosas & $\mathbf{N}$ & $\%$ \\
\hline Omissão de doses & 83 & 83,0 \\
\hline Medicação errada & 12 & 12,0 \\
\hline Hora errada & 3 & 3,0 \\
\hline Dose errada & 2 & 2,0 \\
\hline Total & 100 & 100 \\
\hline
\end{tabular}

Fonte: Dados da pesquisa, 2018

$\mathrm{Na}$ análise, a classe de medicamentos em que ocorreu o maior número de eventos iatrogênicos foram os anti-hipertensivos, com uma frequência de $65(27,0 \%)$ dos eventos. No caso dos antihipertensivos, muitas vezes o paciente apresentava pico hipertensivo, que era constatado através da observação minuciosa dos registros de sinais vitais, no entanto não havia registros da administração da medicação.

Tabela 6. Relação entre a classe de medicamentos e o número de eventos iatrogênicos. Coari, AM

\begin{tabular}{|l|c|c|}
\hline \multicolumn{1}{c}{ Classe de medicamentos } & $\begin{array}{c}\text { N. }{ }^{\circ} \text { Eventos } \\
\text { Iatrogênicos }\end{array}$ & \% \\
\hline Anti-hipertensivo & 65 & 27,0 \\
\hline Antibiótico & 39 & 16,2 \\
\hline Antiácido & 28 & 11,6 \\
\hline Analgésico e antipirético & 27 & 11,2 \\
\hline Anti-inflamatório & 23 & 9,5 \\
\hline Anticolinérgico & 13 & 5,4 \\
\hline Antiemético & 12 & 5,0 \\
\hline Antiplaquetário e Anticoagulante & 11 & 16,0 \\
\hline Antiflatulento & 5 & 2,1 \\
\hline Antimicrobiano & 5 & 2,1 \\
\hline Analgésico potente & 4 & 1,7 \\
\hline Anticonvulsivante & 4 & 1,7 \\
\hline Anti-histamínico & 3 & 1,2 \\
\hline Antimiastênico & 1 & 0,4 \\
\hline Antidiarreico probiótico & 1 & 0,4 \\
\hline \multicolumn{1}{|c|}{ Total } & $\mathbf{2 4 1}$ & $\mathbf{1 0 0}$ \\
\hline
\end{tabular}

Fonte: Dados da pesquisa, 2018

\section{Discussão}

Foram analisados os prontuários de 100 indivíduos internados na clínica médica e diferente de outros estudos, indivíduos do sexo masculino apresentaram maior número relativo em comparação ao sexo feminino, sendo mais da metade das internações (54\%) referentes ao sexo masculino. 
Um estudo demonstrou que os maiores números de internações em um hospital de média a alta complexidade eram de mulheres; no entanto, quando se excluíram as internações por motivo de parto, esse número caiu drasticamente (Castro, Travassos, \& Carvalho, 2002).

Um outro estudo também demonstrou maior prevalência de internação entre indivíduos do sexo feminino em comparação com os do sexo masculino, e que a faixa etária de sessenta anos ou mais apresentou maior frequência de internações (Garbinato, et al., 2007). No entanto, estes estudos não levaram em consideração especificamente a população idosa.

De acordo com a Classificação Internacional de Doenças da $10^{a}$ edição (CID 10), as principais doenças que levaram à internação foram aquelas do aparelho respiratório representadas pela pneumonia, seguida das doenças do aparelho circulatório, diferentemente de outra pesquisa, na qual a principal causa de internação foram as doenças do aparelho circulatório, seguida das doenças do aparelho respiratório (Cuentro, Modesto, Andrade, \& Silva, 2016). A maior parte dos idosos (73\%) apresentava alguma doença preexistente, sendo que 50\% possuíam alguma doença do aparelho circulatório; destes, $48 \%$ possuía Hipertensão Arterial Sistêmica, sendo a doença preexistente mais comum nos idosos analisados, o que corroboraram os estudos de Degani, Júnior, Rodrigues, Luchesi e Marques 2014; Manso, Biffi, e Gerardi, 2015; Nascimento, et al., 2017.

No presente estudo, as principais iatrogenias estavam relacionadas a medicamentos; no entanto, na literatura poucos estudos avaliam com especificidade a iatrogenia medicamentosa, dificultando uma comparação mais precisa. Assim como em outros estudos, foi identificada uma elevada frequência de iatrogenias na população idosa, estando presente nas 100 internações aqui analisadas, totalizando mais de 240 episódios, sendo que, em quatro prontuários, foram identificados mais de dez eventos iatrogênicos que, sob este aspecto, corroboram com outros estudos, apontando que um único paciente pode sofrer múltiplos eventos adversos (Szlejf1, Farfel, Saporetti, Filho, \& Curiati, 2008; Teixeira, Bezerra, Paranaguá, \& Pagotto, 2018). A população idosa tende a apresentar maior gravidade de doença em relação aos jovens; assim, quando internados em hospitais, se tornam mais propensos a eventos adversos (Szlejfl, 2010). Neste estudo, uma das principais iatrogenias, que ocorrem com a população geriátrica, foi com o uso de um grande número de medicamentos (polifarmácia); segundo Lucchetti, Granero, Pires e Gorzoni (2010), a prevalência de polifarmácia é alta em vários setores de atendimento e de atenção à saúde da população.

O tempo de internação e o número de medicamentos utilizados são comumente vistos como fator de risco para a ocorrência de iatrogenias (Szlejf1, Farfel, Saporetti, Filho, \& Curiati, 2008; Teixeira, Bezerra, Paranaguá, \& Pagotto, 2018). Das iatrogenias medicamentosas identificadas, a omissão de doses foi a mais frequente, sendo observada em 83 internações; os anti-hipertensivos foram os medicamentos em que mais ocorreram os eventos iatrogênicos. 
Foram observadas iatrogenias em todos os prontuários analisados neste estudo; no entanto, isso não representa a totalidade, não significa que todos os idosos internados sofram algum tipo de iatrogenia, mas reflete a necessidade de se fazerem mais estudos a respeito desse assunto, buscando compreender suas causas, fatores de risco e pensar em estratégias para diminuir ou minimizar essas ocorrências.

Em todos os prontuários revisados, foi possível concluir que os registros eram pouco detalhados, sem dar destaque à ocorrência das iatrogenias; em apenas um prontuário havia registro explícito das consequências da iatrogenia (paciente alérgico apresentou tremores após administração de dipirona) e, em nenhum prontuário havia medidas para diminuir os efeitos dos eventos iatrogênicos.

Os registros foram redigidos, na maioria das vezes, pelos técnicos de enfermagem, havendo poucos registros ou evoluções redigidas por enfermeiros. Destaca-se que, quando ocorre a subnotificação de iatrogenias, talvez pelo receio de ser punido de alguma forma pela instituição na qual está inserido, ou de ser submetido às sanções éticas e legais, o profissional de enfermagem contribui para outras morbidades, senão para o óbito (Tofoletto, \& Padilha, 2006)

O fator humano é o mais relevante para a ocorrência de iatrogenia; porém, os problemas relacionados à infraestrutura, à falta de insumos e equipamentos, sobrecarga de trabalho, processo de trabalho, absenteísmo e à própria condição clínica do paciente não podem ser desconsiderados. Dessa forma, todas as iatrogenias devem ser investigadas, não com o intuito de punir os culpados, pois o medo da punição leva às subnotificações, mas, sim, com o objetivo de melhorar a assistência prestada (Silva, \& Marziale, 2000; Silva, \& Padilha, 2001).

Assim como em outras pesquisas com abordagem retrospectiva, a escassez de registro mostrou-se como uma das limitações deste estudo.

\section{Conclusão e Considerações Finais}

A partir da análise de 100 prontuários de pacientes idosos hospitalizados, foram encontrados, através da identificação dos registros, 241 iatrogenias medicamentosas. Os eventos iatrogênicos mais frequentes foram o de omissão de doses e a medicação errada.

Os idosos com iatrogenia foram, em sua maioria, homens, com 70 a 79 anos, aposentados, em uso de dez ou mais medicações por dia, com o tempo de internação maior ou igual a dez.

As ações iatrogênicas adquirem maior impacto na população idosa, tendo em vista o processo patológico somado às alterações próprias do processo de envelhecimento. 
Os dados presentes neste estudo demonstram que as iatrogenias medicamentosas fazem parte da prática da equipe de saúde e ocorrem com frequência. Importa registrar que a equipe de saúde deve procurar identificar as causas das iatrogenias, os fatores de risco e as suas principais complicações e, juntamente com as instituições hospitalares, promover capacitação da equipe e instituir protocolos que reduzam a ocorrência de eventos iatrogênicos.

Encontrar estudos que abordem as iatrogenias sob diferentes aspectos e com o uso de diferentes metodologias, na sua maioria prospectivas, ou baseadas em relatos dos profissionais, dificultou a comparação dos resultados com este estudo; no entanto, percebe-se a carência de estudos que abordem as iatrogenias especificamente em pacientes idosos. Apesar da importância dos resultados, problemas metodológicos relacionados ao tamanho da amostra, a escassez dos registros se mostraram como limitações para esta pesquisa. No entanto, demonstram a necessidade de mais estudos que busquem compreender as causas das iatrogenias e promovam medidas que reduzam ou minimizem as ocorrências iatrogênicas.

\section{Referências}

Carvalho-Filho, E. T., Saporetti L., Souza, M. A., Arantes, A. C., Vaz, M. Y., Hojaiji, N. H., Alencar, Y. M., \& Curiati, J. E. (1998). Iatrogenia em pacientes idosos hospitalizados. Rev Saúde Pública, 32(1), 36-42. Recuperado em 18 agosto, 2017, de: http://www.scielo.br/scielo.php?pid=S003489101998000100005\&script=sciabstract\&tlng=pt .

Castro, M. S., Travassos, S., Carvalho, M. S. (2002). Fatores associados a internações hospitalares no Brasil. Rev Ciência e Saúde Coletiva, 7(4), 795-811. Recuperado em 18 agosto, 2017, de: http://www.scielo.br/scielo.php?script=sci_abstract\&pid=S1413-81232002000400014\&lng= es\&nrn=iso\&tlng=pt\&userID=-2

Correr, C. J., Pontarolo, R., Ferreira, L. C., \& Baptistão, S. A. (2007). Riscos de problemas relacionados com medicamentos em pacientes de uma instituição geriátrica. Rev Brasileira de Ciências Farmacêuticas, 43(1), 55-62. Recuperado em 15 setembro, 2017, de: http://www.scielo.br/pdf/rbcf/v43n1/06.pdf.

Cuentro, V., Modesto, T., Andrade, M., \& Silva, M. (2016). Prevalência e fatores associados à polifarmácia entre idosos de um hospital público. Rev. Contexto e Saúde, 16(30), 28-35. Recuperado em 01 dezembro, 2017, de: https://www.revistas.unijui.edu.br/ index.php/contextoesaude/article/view/4448.

Degani, G. C., Júnior, G. A., Rodrigues, R. A., Luchesi, B. M., \& Marques, S. (2014). Idosos vítimas de trauma: doenças preexistentes, medicamento em uso no domicílio e índices de trauma. Rev. Bras. Enferm., 67(5), 759-765. Recuperado em 01 dezembro, 2017, de: https://www.google.com/url?sa=t\&source=web\&rct=j\&url=http://www.scielo.br/pdf/reben/vv67n5/ 0034-7167-reben-67-05-0759.pdf\&ved=2ahUKEwimrI3o2PniAhVIILKGHStXDIQQFj ABegQIBRAB\&usg=AOvVaw2RXdIEWAMQXm1vKdmsKcUG. 
Diniz, E. A. (2010). Iatrogenia medicamentosa em idosos: análise da equipe de saúde São João II de Conselheiro Lafaiete. Trabalho de Conclusão de Curso de Especialização em Atenção Básica em Saúde da Família. Universidade Federal de Minas Gerais. Recuperado em 30 agosto, 2017, de: https://www.nescon.medicina.ufmg.br/biblioteca/registro/_Iatrogenia_medicamentosa_em_idosos_ _analise_da_equipe_de_saude_Sao_Joao_II_de_Conselheiro_Lafaiete_/458.

Garbinato, L. R., Béria, J. U., Figueiredo, A. C. L., Raymann, B., Gigante, L. P., Palazzo, L. dos S., \& Aerts, D. R. G. de C. (2007). Prevalência de internação hospitalar e fatores associados: um estudo de base populacional em um centro urbano no Sul do Brasil. Cad. Saúde Pública 23(1), 217-224. Recuperado em 01 dezembro, 2017, de: http://www.scielo.br/scielo.php?pid=S0102311X2007000100023\&script=sci_abstract\&tlng=pt.

Guiselli, R. S., Ely, L. S., Engroff, P., Nogueira, E. L., \& Gomes, I. (2016). Estudo do uso de medicamentos potencialmente inapropriados em idosos da Estratégia Saúde da Família. Rev KairósGerontologia, 19(2), 243-257. Recuperado em 20 outubro, 2017, de: https://revistas.pucsp.br/kairos/article/view/32355/22368.

Lucchetti, G., Granero A., Pires, S., \& Gorzoni, M. (2010). Fatores associados à polifarmácia em idosos institucionalizados. Rev. Bras. Geriatr. Gerontol., 13(1), 51-58. Recuperado em 20 outubro, 2017, de: http://dx.doi.org/10.1590/S1809-98232010000100006.

Madalosso, A. R. (2000). Iatrogenia no cuidado de enfermagem: dialogando com o perigo no quotidiano profissional. Rev Latino-Am de Enfermagem, 8(3), 11-17. Recuperado em 18 agosto, 2017. de: http://www.scielo.br/scielo.php?pid=S010411692000000300003\&script=sci_ abstract\&tlng=pt.

Manso, M. A., Biffi, E. C., \& Gerardi, T. J. (2015). Prescrição inadequada de medicamentos a idosos portadores de doenças crônicas em um plano de saúde do município de São Paulo, Brasil. Rev. Bras. Geriatr. Gerontol., 18(1), 151-164. Recuperado em 01 dezembro, 2017, de: http://dx.doi.org/10.1590/1809-9823.2015.14056.

Matos, L., Schmidt, A., Peserico, A., Moreira, C., Fernandes, F., Carlos, G., Aguirre, M., \& Souza, M. (2011). A ação iatrogênica da equipe de enfermagem para a saúde do idoso. Rev Contexto e Saúde, 10(20), 541-544. Recuperado em 18 agosto, 2017, de: https://www.revistas.unijui.edu.br/index.php/contextoesaude/article/view/1575/1330.

Nascimento, R. C. R. M. do, Álvares, J., Guerra Junior, A. A., Gomes, I. C., Silveira, M. R., Costa, E. A., Leite, S. N., Costa, K. S., Soeiro, O. M., Guibu, I. A., Karnikowski, M. G. de O., \& Acurcio, F. de A. (2017). Polifarmácia: uma realidade na atenção primária do Sistema Único de Saúde. Rev. Saúde Pública, 51(2), 1s-12s. Recuperado em 01 fevereiro, 2018, de: http://www.scielo.br/pdf/rsp/v51s2/pt_0034-8910-rsp-S1518-51-s2-87872017051007136.pdf.

Padilha, K. G. (2001). Ocorrências iatrogênicas na UTI e o enfoque de qualidade. Rev Latino-Am de Enfermagem, 9(5), 91-96. Recuperado em 18 agosto, 2017, de: https://www.revistas.usp.br/rlae/article/viewFile/1606/1651.

Padilha, K. G. (2006). Ocorrências iatrogênicas em unidade de terapia intensiva (UTI): análise dos fatores relacionados. Rev Paulista de Enfermagem, 25(1),18-23. Recuperado em 01 julho, 2018, de: https://repositorio.usp.br/item/001564403.

Schmidt, E. A., Valle, D. A., Martins, J. S., Borges, J. L., Júnior, S. L., \& Ribeiro, U. R. (2011). Iatrogenia como desdobramento da relação médico-paciente. Rev Bras Clin Med., 9(3), 146-149. Recuperado em 20 outubro, 2017, de: http://files.bvs.br/upload/S/1679-1010/2011/v9n2/a1827.pdf.

Silva, D. M., Marziale, M. H. (2000). Absenteísmo de trabalhadores de enfermagem em um hospital universitário. Rev Lat Americ Enferm., 8(5), 44-51. Recuperado em 20 outubro, 2017, de: http://www.scielo.br/scielo.php?script=sci_abstract\&pid=S0104-11692000000500007\&lng= pt\&nrm=iso. 
Silva, S. C., \& Padilha, K. G. (2001). Parada cardiorrespiratória na unidade de terapia intensiva: considerações teóricas sobre os fatores relacionados às ocorrências de iatrogênicas. Rev Esc Enferm USP., 35(4), 360-365. Recuperado em 20 outubro, 2017, de: http://www.scielo.br/pdf/reeusp/v35n4/v35n4a07.pdf.

Szlejf, C. (2010). Eventos adversos médicos em idosos hospitalizados: frequência e fatores de risco em enfermaria de geriatria. Tese de doutorado em Ciências. Faculdade de Medicina, Universidade de São Paulo. Recuperado em 01 julho, 2017, de: https://www.teses.usp.br/teses/disponiveis/5/5144/tde-30112010-152956/publico/ ClaudiaSzlejf.pdf.

Szlejf, C., Farfel, J. M., Saporetti, L. A., Filho, W. J., \& Curiati, J. A. (2008). Fatores relacionados com a ocorrência de iatrogenia em idosos internados em clínica geriátrica: estudo prospectivo. Rev Einstein, 6(3), 337-342. Recuperado em 21 outubro, 2017, de: http://apps.einstein.br/revista/arquivos/PDF/966-v6n3aA0966portp337-42.pdf.

Teixeira, C. C., Bezerra, A. L., Paranaguá, T. T. de B., \& Pagotto, V. (2018). Fatores relacionados à ocorrência de eventos adversos em pacientes idosos internados. Rev Baiana Enferm., 32, e25772. Recuperado em 01 julho, 2018, de: DOI 10.18471/rbe.v32.25772.

Tofoletto, M. C., \& Padilha, K. C. (2006). Consequências de erros de medicação em unidades de terapia intensiva e semi-intensiva. Rev Esc Enferm USP, 40(2), 247-252. Recuperado em 01 julho, 2017, de: http://www.scielo.br/pdf/reeusp/v40n2/12.pdf.

Recebido em 28/06/2019

Aceito em 30/09/2019

Andriele Valentim da Costa - Graduanda do curso de enfermagem, Universidade Federal do Amazonas, Instituto de Saúde e Tecnologia/UFAM/ISB. Coari. Amazonas, Brasil.

E-mail: andriele.vcosta@gmail.com

Luana de Melo Lisboa - Graduanda do curso de enfermagem, Universidade Federal do Amazonas, Instituto de Saúde e Tecnologia/UFAM/ISB. Coari, Amazonas, Brasil.

E-mail: luanamelo.lisboa@gmail.com

Deyvylan Araujo Reis - Professor Doutor do curso de graduação em enfermagem, Universidade Federal do Amazonas, Instituto de Saúde e Tecnologia/UFAM/ISB. Coari, Amazonas, Brasil.

E-mail: deyvylan@hotmail.com 
Hyana Kamila Ferreira de Oliveira - Mestranda do Programa de Pós-Graduação em Enfermagem, Universidade Federal do Amazonas. Professora do Curso de Graduação em Enfermagem, Universidade Federal do Amazonas, Instituto de Saúde e Tecnologia/UFAM/ISB. Coari, Amazonas, Brasil.

E-mail: hyanakamila@hotmail.com 